\title{
O impacto do uso de métodos contraceptivos na adolescência: uma revisão
}

\section{integrativa}

\author{
The impact of using contraceptive methods in adolescence: an integrative review \\ El impacto del uso de anticonceptivos en la adolescencia: una revisión integradora
}

Recebido: 19/01/2022 | Revisado: 27/01/2022 | Aceito: 29/01/2022 | Publicado: 30/01/2022

\author{
Isabela Almeida Alves \\ ORCID: https://orcid.org/0000-0003-4979-2259 \\ Universidade Ceuma, Brasil \\ E-mail: isabelaalmeidaalves@outlook.com \\ Victória Caroline Alves Ferreira \\ ORCID: https://orcid.org/0000-0002-6046-6529 \\ Universidade Ceuma, Brasil \\ E-mail: viccaroline18@gmail.com \\ Karyne Gleyce Zemf Oliveira \\ ORCID: https://orcid.org/0000-0002-3676-7719 \\ Universidade Ceuma, Brasil \\ E-mail: Karyne_zemf@hotmail.com \\ Mónica Andréa Miranda Aragão \\ ORCID: https://orcid.org/0000-0002-7689-9070 \\ Universidade Ceuma, Brasil \\ E-mail: monicamirandaaragao@gmail.com
}

\begin{abstract}
Resumo
Objetivo: Descrever fatores que influenciam o uso de contraceptivos por adolescentes. Metodologia: Trata-se de uma revisão integrativa referente ao uso de anticoncepcionais na adolescência. A pesquisa foi realizada em junho de 2021 nas seguintes bases de dados: MEDLINE, LILACS e PubMed. Para realização da revisão utilizou-se descritores determinados com base nos Descritores em Ciências da Saúde (DeCS), foram eles: "adolescentes", "anticoncepcionais", "contraceptivos", "teenager" e "contraception". Foram encontrados 143 artigos, entretanto, após critérios de inclusão e exclusão foram selecionados 8 artigos. Resultado: É visto que mesmo na contemporaneidade, há ainda uma gama de fatores que dificultam o uso de métodos contraceptivos por adolescentes. Fatores como cultura, religião, faixa etária, desigualdade de gênero e a classe social são determinantes na escolha de um método de proteção. Além disso, a falta de comunicação com os pais, influenciou a falta de conhecimento dos jovens sobre os métodos, assim como limitou a busca de atendimento profissional no sistema de saúde e a adesão. Conclusão: Embora a educação sexual, o sigilo médico e o acesso a métodos contraceptivos, sejam direitos garantidos aos adolescentes, muitos desses ainda não possuem acesso ao planejamento familiar. Isso acaba colocando muitos adolescentes em um estado de vulnerabilidade se tratando de IST's e gravidez precoce.
\end{abstract}

Palavras-chave: Adolescentes; Anticoncepcionais; Contraceptivos.

\begin{abstract}
Objective: To describe factors that influence the use of contraceptives by adolescentes. Methodology: This is an integrative review regarding the use of contraceptives in adolescence. The research was conducted in June 2021 in the following databases: MEDLINE, LILACS and PubMed. To perform the review, we used descriptors determined based on the Descriptors in Health Sciences (DeCS), they were: "adolescents", "contraceptives", "contraceptives", "teenager" and "contraception". A total of 143 articles were found, however, after inclusion and exclusion criteria, 8 articles were selected. Result: It is seen that even in contemporary times, there are still a range of factors that hinder the use of contraceptive methods by adolescents. Factors such as culture, religion, age group, gender inequality and social class are determinant in choosing a method of protection. In addition, the lack of communication with parents influenced the lack of knowledge of young people about the methods, as well as limited the search for professional care in the health system and support. Conclusion: Although sex education, medical secrecy and access to contraceptive methods, rights guaranteed to adolescents, many of them do not yet have access to family planning. This ends up putting many teenagers in a state of vulnerability when it comes to STIs and early pregnancy.
\end{abstract}

Keywords: Teenager; Contraceptive agents; Contraception; Contraceptive.

\section{Resumen}

Objetivo: Describir los factores que influyen en el uso de anticonceptivos por parte de los adolescentes. Metodología: Se trata de una revisión integradora sobre el uso de anticonceptivos en la adolescencia. La investigación se realizó en junio de 2021 en las siguientes bases de datos: MEDLINE, LILACS y PubMed. Para realizar la revisión, se utilizaron 
descriptores determinados en base a los Descriptores en Ciencias de la Salud (DeCS), que fueron: "adolescentes", "anticonceptivos", "anticonceptivos", "adolescente" y "anticoncepción". ". Se encontraron un total de 143 artículos, sin embargo, después de los criterios de inclusión y exclusión, se seleccionaron 8 artículos. Resultado: Se ve que incluso en los tiempos contemporáneos, todavía hay una serie de factores que dificultan el uso de métodos anticonceptivos por parte de los adolescentes. Factores como la cultura, la religión, el grupo de edad, la desigualdad de género y la clase social son determinantes a la hora de elegir un método de protección. Además, la falta de comunicación con los padres influyó en la falta de conocimiento de los jóvenes sobre los métodos, así como limitó la búsqueda de atención profesional en el sistema de salud y apoyo. Conclusión: Aunque la educación sexual, el secreto médico y el acceso a métodos anticonceptivos, derechos garantizados a los adolescentes, muchos de ellos aún no tienen acceso a la planificación familiar. Esto termina poniendo a muchas adolescentes en un estado de vulnerabilidad cuando se trata de ITS y embarazo temprano.

Palabras clave: Adolescentes; Anticonceptivos; Contraconceptivo.

\section{Introduçãa}

A adolescência é compreendida como a faixa etária que vai dos 10 aos 19 anos completos, sendo a pré-adolescência dos 10 aos 14 anos e a adolescência propriamente dita dos 15 aos 19 anos (Brasil, 1990). Esse período é marcado pela puberdade, fase caracterizada pelo aumento da amplitude dos pulsos de secreção dos hormônios folículo-estimulante (FSH) e hormônio luteinizante (LH), o que implica na secreção dos hormônios gonadais; o estrogênio e a progesterona no sexo feminino e a testosterona no sexo masculino. Essas alterações levam ao desenvolvimento das características sexuais secundárias e a descoberta da sexualidade (Campos Júnior \& Burns, 2014).

Nessa fase ocorre a consolidação da identidade de gênero e sexo, que nem sempre acompanha o amadurecimento afetivo e cognitivo. Apesar de ser natural para a faixa etária, isso torna o adolescente vulnerável a certos riscos, principalmente quando se trata do início das práticas sexuais. Por isso, é essencial a utilização de métodos contraceptivos por essa população, com a finalidade de evitar uma gravidez não planejada e a disseminação de infecções sexualmente transmissíveis (IST's) (Alves \& Lopes, 2008; Ezenwak, et al., 2020).

Os principais métodos utilizados pelos adolescentes são os preservativos e a pílula, de forma combinada ou não. A escolha desses métodos em específico se dá pela facilidade de aquisição, tendo em vista o baixo custo, além da falta de conhecimento acerca dos outros disponíveis. Isso retrata a falha do processo educativo, tanto na esfera escolar, que peca no ensino sobre saúde sexual e reprodutiva, quanto da esfera pública, que incentiva largamente o uso de preservativos, mas não apresenta investimentos semelhantes para a instrução acerca de outros métodos (Sserwanja, et al., 2020).

A possibilidade de ocorrência de efeitos adversos que alguns métodos podem causar, em especial os hormonais, pode deixar os adolescentes receosos com seu uso, levando a pouca adesão, e por vezes desistência (Sserwanja, et al., 2020). Além disso, o desconforto e a irregularidade do método, além da falta de informação sobre o uso correto dos mesmos, abrem possibilidade para eventuais falhas que comumente são associadas erroneamente a eficácia dos anticoncepcionais (Vieira, et al., 2006).

A importância do esclarecimento dessa temática foi exemplificada em um estudo realizado no Brasil, no qual o parto constituiu a principal causa de internação de adolescentes do sexo feminino no SUS. Na faixa etária de 15 a 19 anos, o motivo predominante de internação das mulheres foi inerente à gravidez, parto, pós-parto e as complicações associadas (Dadoorian, 2003).

Nesse ínterim, pode-se afirmar que a educação sexual, especialmente voltada para o uso de contraceptivos, é de grande valia no cenário de saúde atual, uma vez que além do grande impacto que uma gravidez ou IST pode causar na vida do adolescente, há ainda o ônus que é gerado para o sistema de saúde. As consequências desencadeadas por uma gestação ou uma infecção incluem, ainda, o abandono dos estudos ou o seu adiamento, maior dependência econômica dos pais, visto que a maioria das jovens continua morando com os pais após o nascimento do filho, já que o pai da criança é, na maioria dos casos, também adolescente (U.S. Department of Health and Human Services, 2018). 
Com isso, o objetivo do presente estudo foi averiguar o uso de métodos contraceptivos entre os adolescentes, os fatores que influenciam sua utilização ou não e o impacto que esses causam na vida dos usuários mais jovens.

\section{Metodologia}

Trata-se de uma revisão integrativa da literatura, na busca de evidências científicas sobre o uso de métodos contraceptivos por adolescentes. Vale ressaltar que esse tipo de pesquisa direciona o conhecimento atualizado sobre o tema proposto, com o objetivo de identificar, avaliar e resumir os resultados das pesquisas independentes (Souza, et. al., 2010).

Para a coleta de dados, utilizou-se a seguinte sucessão de etapas: seleção do tema; estabelecimento da pergunta norteadora: "quais fatores influenciam a utilização ou não dos anticoncepcionais pelos adolescentes?”; definição de critérios de inclusão e exclusão; levantamento bibliográfico com seleção de artigos a partir dos critérios definidos; análise dos estudos e interpretação dos resultados e, por fim, apresentação da revisão e dos dados obtidos.

A coleta de dados foi realizada em novembro de 2021 nas seguintes bases de dados: Medical Literature Analysis and Retrieval System Online (MEDLINE) e Literatura Latino-Americana e do Caribe em Ciências da Saúde (LILACS). Para o levantamento bibliográfico utilizou-se descritores determinados com base nos Descritores em Ciências da Saúde (DeCS), foram eles: "adolescentes", "anticoncepcionais", "contraceptivos", "teenager" e "contraception". Empregou-se o operador booleano AND e OR nas seguintes combinações: "adolescentes" OR "teenager" AND “contraceptivos" OR "contraception".

Os critérios de inclusão utilizados foram artigos publicados nos últimos 3 anos, em português e inglês e que respondessem à pergunta norteadora. Não houve delineamento quanto ao tipo de estudo dos artigos, sendo considerados todos os tipos de pesquisa. Foram utilizados os filtros: Texto Completo Disponível, e como assunto principal: Anticoncepcionais Orais, Anticoncepção, Anticoncepcionais, Adolescente e Comportamento Sexual.

A partir disso, foram identificados 143 artigos nas buscas empregadas nas bases de dados mencionadas, dos quais 100 foram excluídos após a leitura de seus resumos por não responderem à pergunta norteadora. Após a leitura na íntegra dos 43 artigos restantes, apenas 8 foram selecionados por contemplar o objetivo da pesquisa e estão dispostos no Quadro 1.

\section{Resultados e Discussão}

Dos artigos selecionados, seis tratavam-se de pesquisa transversal, um de estudo exploratório e um de pesquisa demográfica e de saúde.

Seis desses abordaram o conhecimento e a aderência das adolescentes ao uso de métodos contraceptivos, um deles abordou apenas os fatores que limitam a utilização de contraceptivos pelos mais jovens e um deles discorreu sobre a qualidade de vida das adolescentes que utilizaram métodos hormonais, observando seus impactos na pressão arterial, na densidade óssea e na saúde mental, conforme mostra o Quadro 1.

Quadro 1 - Distribuição dos artigos publicados nos anos de 2019 a 2021 conforme autor, ano, periódico, tipo de estudo, população e amostra, objetivos, resultados e conclusão. Imperatriz/MA, 2021.

\begin{tabular}{|c|c|c|c|c|c|c|}
\hline $\begin{array}{c}\text { AUTOR } \\
\text { / ANO }\end{array}$ & PERÍODICO & $\begin{array}{l}\text { TIPO DE } \\
\text { ESTUDO }\end{array}$ & $\begin{array}{l}\text { POPULAÇÃO/ } \\
\text { AMOSTRA }\end{array}$ & OBJETIVO & RESULTADOS & CONCLUSÃO \\
\hline $\begin{array}{l}\text { Ganle } \text { et } \\
\text { al (2019) }\end{array}$ & $\begin{array}{c}\text { International } \\
\text { Journal for } \\
\text { Equity in } \\
\text { Health }\end{array}$ & $\begin{array}{c}\text { Estudo } \\
\text { quantitativ } \\
\text { a com } \\
\text { abordagem } \\
\text { transversal }\end{array}$ & $\begin{array}{l}\text { Foram } \\
\text { entrevistadas } 242 \\
\text { adolescentes } \\
\text { refugiadas, } \\
\text { residentes no } \\
\text { campo de } \\
\text { Budumburam, } \\
\text { com idades entre }\end{array}$ & $\begin{array}{l}\text { Avaliar o } \\
\text { comportamento } \\
\text { sexual e uso de } \\
\text { anticoncepcionais } \\
\text { entre mulheres } \\
\text { adolescentes } \\
\text { refugiadas em } \\
\text { Gana. }\end{array}$ & $\begin{array}{l}\text { Os resultados mostraram que cerca de } \\
64,5 \% \text { das entrevistadas já tinha ouvido } \\
\text { falar sobre anticoncepcionais, sendo o } \\
\text { preservativo masculino o mais conhecido, } \\
54,8 \% \text {, seguido da pílula, } 22,8 \% \text { e dos } \\
\text { implantes, } 19,2 \% \text {. No entanto, } 38,5 \% \text { das } \\
\text { adolescentes não sabiam os locais onde } \\
\text { poderiam obter os anticoncepcionais }\end{array}$ & $\begin{array}{l}\text { Tanto a conscientização } \\
\text { quanto o uso de } \\
\text { contraceptivos modernos } \\
\text { entre } r \text { adolescentes } \\
\text { refugiadas do sexo } \\
\text { feminino são muito } \\
\text { menores do que as taxas } \\
\text { entre adolescentes não }\end{array}$ \\
\hline
\end{tabular}




\begin{tabular}{|c|c|c|c|c|c|c|}
\hline & & & 14-19 anos. & & $\begin{array}{l}\text { modernos. Apenas 7,3\% delas relataram } \\
\text { usar um método contraceptivo atualmente. } \\
\text { Fatores como idade, educação sexual em } \\
\text { casa, na escola ou na igreja, a realização } \\
\text { da atividade sexual bêbada e a quantidade } \\
\text { de parceiros, influenciaram a utilização da } \\
\text { contracepção. }\end{array}$ & $\begin{array}{lr}\text { refugiadas em } & \text { Gana, } \\
\text { levantando } & \text { questões } \\
\text { sobre equidade, justiça } \\
\text { de saúde global e } \\
\text { os dobre } \\
\text { reprodutiva de } & \text { saúde } \\
\text { adolescentes que vivem } \\
\text { em situação } \\
\text { refugiadas. }\end{array}$ \\
\hline $\begin{array}{l}\text { Agyema } \\
\text { ng et al } \\
(2019)\end{array}$ & $\begin{array}{c}\text { PanAfrican } \\
\text { Medical } \\
\text { Journal }\end{array}$ & $\begin{array}{c}\text { Estudo } \\
\text { transversal } \\
\text { com } \\
\text { abordagem } \\
\text { quantitativ } \\
\text { a }\end{array}$ & $\begin{array}{l}\text { Foram recrutadas } \\
\text { um total de } 200 \\
\text { adolescentes do } \\
\text { sexo feminino } \\
\text { entre as idades de } \\
16-19 \text { anos, } \\
\text { identificadas } \\
\text { como sexualmente } \\
\text { ativas, residentes } \\
\text { no distrito de } \\
\text { Atwima } \\
\text { Kwanwoma por } \\
\text { pelo menos 6 } \\
\text { meses. }\end{array}$ & $\begin{array}{l}\text { Buscou entender a } \\
\text { magnitude e os } \\
\text { fatores associados } \\
\text { que influenciam o } \\
\text { uso de } \\
\text { anticoncepcionais } \\
\text { entre as } \\
\text { adolescentes no } \\
\text { distrito de Atwima } \\
\text { Kwanwoma. }\end{array}$ & $\begin{array}{l}\text { A maioria das participantes }(95,0 \%) \\
\text { tinham conhecimentos sobre os métodos } \\
\text { contraceptivos e seus benefícios. } \\
\text { Observou-se como método } \\
\text { anticoncepcional mais conhecido o } \\
\text { preservativo, e também mais usado (30\%), } \\
\text { seguido do injetável }(25,0 \%) \text {, da pílula } \\
(11,1 \%) \text {, enquanto poucos }(5,0 \%) \\
\text { conheciam o DIU. Porém, } 82 \% \text { das } \\
\text { entrevistadas não } \\
\text { anticoncepcionais. Os fatores que } \\
\text { influenciaram o uso de contraceptivos } \\
\text { foram os efeitos colaterais, a falta de } \\
\text { conhecimento em relação ao local de } \\
\text { aquisição desses, práticas culturais, a } \\
\text { ridicularização pelos colegas e a não } \\
\text { discussão da saúde reprodutiva em casa. }\end{array}$ & $\begin{array}{l}\text { O estudo revelou que } \\
\text { mesmo com a alta taxa } \\
\text { de conhecimento acerca } \\
\text { dos anticoncepcionais } \\
\text { entre as adolescentes, } \\
\text { apenas } 18 \% \text { delas faziam } \\
\text { o uso dos mesmos. } \\
\text { Sendo o preservativo o } \\
\text { método mais utilizado. E } \\
\text { o fator principal } \\
\text { identificado para } \\
\text { justificar essa baixa } \\
\text { adesão foram os efeitos } \\
\text { colaterais. }\end{array}$ \\
\hline $\begin{array}{c}\text { Ezenwak } \\
\text { a } \text { et al } \\
\text { (2020) }\end{array}$ & $\begin{array}{c}\text { BMC Public } \\
\text { Health }\end{array}$ & $\begin{array}{c}\text { Estudo } \\
\text { exploratóri } \\
\text { o com } \\
\text { abordagem } \\
\text { quantitativ } \\
\text { a }\end{array}$ & $\begin{array}{l}\text { Seis comunidades } \\
\text { no estado de } \\
\text { Ebonyi, sudeste } \\
\text { da Nigéria. Os } \\
\text { entrevistados } \\
\text { incluíram } \\
\text { formuladores de } \\
\text { políticas, líderes } \\
\text { comunitários, } \\
\text { saúde } \\
\text { prestadores de } \\
\text { serviços e pais de } \\
\text { adolescentes. }\end{array}$ & $\begin{array}{c}\text { Examinar a } \\
\text { percepção de } \\
\text { influenciadores e } \\
\text { dos responsáveis } \\
\text { pelos adolescentes } \\
\text { sobre os fatores } \\
\text { que restringem a } \\
\text { utilização de } \\
\text { serviços } \\
\text { anticoncepcionais } \\
\text { entre adolescentes } \\
\text { no estado de } \\
\text { Ebonyi, Nigéria. }\end{array}$ & $\begin{array}{l}\text { Foram observados fatores que impediam a } \\
\text { utlização de contraeptivos pela } \\
\text { adolescentes. Dentre eles, as de nível } \\
\text { individual, como a falta de conhecimento, } \\
\text { os efeitos colaterais, o custo, a timidez e a } \\
\text { baixa autoestima. Barreiras relacionadas a } \\
\text { familia, como a falta de diálogo com os } \\
\text { pais. Barreiras dos sistemas de saúde, } \\
\text { como a atitude hostil dos profissionais da } \\
\text { saúde, falta de confidencialidade e fraco } \\
\text { apoio instuticional de centros voltados } \\
\text { para jovens. Normas culturais, religiosas e } \\
\text { de gênero. Fatores sociais mais amplos, } \\
\text { como influência de pares e da mídia, } \\
\text { ausência de educação sexual nas escolas, } \\
\text { falta de redes sociais nas comunidades; } \\
\text { também foram percebidos como limitantes } \\
\text { do acesso a anticoncepcionais para } \\
\text { adolescentes. }\end{array}$ & 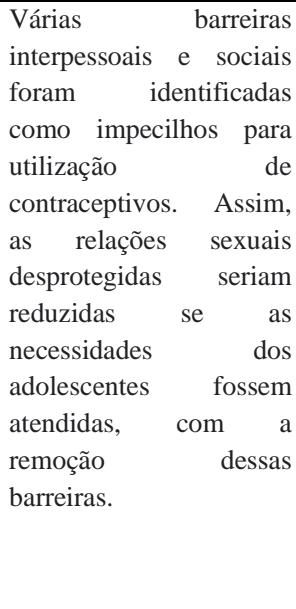 \\
\hline $\begin{array}{c}\text { Klara } \\
\text { Lewando } \\
\text { wski, } \\
\text { Duttge e } \\
\text { Meyer } \\
(2020)\end{array}$ & $\begin{array}{c}\text { Quality of Life } \\
\text { Research }\end{array}$ & $\begin{array}{c}\text { Pesquisa } \\
\text { transversal } \\
\text { com } \\
\text { abordagem } \\
\text { quantitativ } \\
\text { a. }\end{array}$ & $\begin{array}{l}\text { A presente análise } \\
\text { foi baseada em } \\
1695 \text { participantes } \\
\text { do sexo feminino, } \\
\text { de } 15 \text { a } 17 \text { anos de } \\
\text { idade. }\end{array}$ & $\begin{array}{c}\text { Investigar se a } \\
\text { contracepção } \\
\text { hormonal em } \\
\text { adolescentes de } 15 \\
\text { a } 17 \text { anos estava } \\
\text { ligada à qualidade } \\
\text { de vida, } \\
\text { relacionada à } \\
\text { saúde e a } \\
\text { problemas de } \\
\text { saúde mental. }\end{array}$ & $\begin{array}{l}\text { A pressão arterial média calculada das } \\
\text { adolescentes que faziam uso de } \\
\text { anticoncepcionais hormonais foi cerca } 1,7 \\
\text { mmHg maior do que nas não } \\
\text { usuárias. Além disso, os níveis séricos de } \\
25 \text { (OH) D foram significativamente } \\
\text { maiores em usuárias de anticoncepcionais } \\
\text { orais do que em não usuárias. Observou-se } \\
\text { que a taxa de prescrição de medicamentos } \\
\text { psicotrópicos era maior para meninas que } \\
\text { tomavam pílulas anticoncepcionais orais } \\
\text { (1,4\%) em comparação com aquelas que } \\
\text { não usavam contracepção hormonal, mas } \\
\text { os indicadores psicométricos de bem-estar } \\
\text { mental foram semelhantes nos dois } \\
\text { grupos. }\end{array}$ & 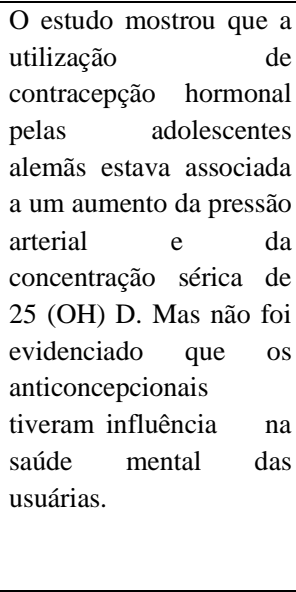 \\
\hline $\begin{array}{c}\text { Bakesii } \\
\text { ma et al } \\
(2020)\end{array}$ & $\begin{array}{c}\text { Reproductive } \\
\text { Health }\end{array}$ & $\begin{array}{c}\text { Estudo } \\
\text { transversal } \\
\text { com } \\
\text { abordagem } \\
\text { quantitativ } \\
\text { a }\end{array}$ & $\begin{array}{l}\text { Um total de } 839 \\
\text { adolescentes } \\
\text { refugiadas, } \\
\text { que eram } \\
\text { sexualmente } \\
\text { ativas ou em } \\
\text { união estável } \\
\text { foram inscritas }\end{array}$ & $\begin{array}{l}\text { Determinar a } \\
\text { prevalência e os } \\
\text { fatores associados } \\
\text { ao uso de } \\
\text { anticoncepcionais } \\
\text { modernos entre } \\
\text { adolescentes } \\
\text { refugiadas no }\end{array}$ & $\begin{array}{l}\text { Os resultados apontaram que } 90,3 \% \text { das } \\
\text { participantes tinham algum conhecimento } \\
\text { acerca dos anticoncepcionais. A } \\
\text { prevalência do uso de contraceptivos entre } \\
\text { as adolescentes foi de } 8,7 \% \text {. Dessas, } \\
42,5 \% \text { faziam uso de injetáveis. O } \\
\text { principal fator apontado }(39,3 \%) \text { para a } \\
\text { não adesão aos contraceptivos foi o medo }\end{array}$ & $\begin{array}{l}\text { Menos de um décimo } \\
\text { das participantes faziam } \\
\text { uso de contraceptivos, } \\
\text { assim, a maior parte das } \\
\text { adolescentes estavam } \\
\text { vulneráveis a gravidez } \\
\text { precoce. Os principais } \\
\text { motivos para o não uso }\end{array}$ \\
\hline
\end{tabular}




\begin{tabular}{|c|c|c|c|c|c|c|}
\hline & & & $\begin{array}{l}\text { consecutivamente } \\
\text { com idades entre } \\
10 \text { e } 19 \text { anos. }\end{array}$ & norte de Uganda. & $\begin{array}{l}\text { dos efeitos colaterais. A idade, estado } \\
\text { civil, número de filhos vivos, idade do } \\
\text { companheiro, ocupação e a escolaridade } \\
\text { também foram fatores associados ao uso } \\
\text { de contraceptivos. }\end{array}$ & $\begin{array}{l}\text { foi o medo dos efeitos } \\
\text { colaterais, proibição do } \\
\text { parceiro e a falta de } \\
\text { conhecimento. }\end{array}$ \\
\hline $\begin{array}{l}\text { Crawfoa } \\
\quad \text { rd, } \\
\text { Atchinso } \\
\text { n, Ajayi, } \\
\text { Doyle } \\
(2021)\end{array}$ & $\begin{array}{c}\text { Reproductive } \\
\text { Health }\end{array}$ & $\begin{array}{l}\text { Estudo } \\
\text { transversal } \\
\text { com } \\
\text { abordagem } \\
\text { quantitativ } \\
\quad \text { a. }\end{array}$ & $\begin{array}{l}\text { Meninas solteiras } \\
\text { de } 15 \text { a } 19 \text { anos do } \\
\text { estado de Ogun, } \\
\text { Nigéria. }\end{array}$ & $\begin{array}{l}\text { O objetivo foi } \\
\text { identificar o uso } \\
\text { de } \\
\text { anticoncepcionais } \\
\text { modernos entre } \\
\text { meninas solteiras } \\
\text { de } 15 \text { a } 19 \text { anos. }\end{array}$ & $\begin{array}{l}\text { Observou-se que a maioria das } \\
\text { intrevistadas já tinham ouvido falar de } \\
\text { contracepção. Cerca de } 63,2 \% \text { das } \\
\text { adolescentes sexualmente ativas usavam } \\
\text { algum método contraceptivo. O método } \\
\text { mais utilizado foi o preservativo } \\
\text { masculino (50,3\%). A pilula de } \\
\text { emergência foi o segundo mais usado } \\
(16,7 \%) \text {. Os fatores que incluenciaram a } \\
\text { utilização de contracepção foram a idade, } \\
\text { escolaridade, apoio do parceiro e dos } \\
\text { amigos, morar em uma área urbana, nível } \\
\text { socioeconômico, número de gestações e } \\
\text { acesso a mídia e a serviços de saúde. }\end{array}$ & $\begin{array}{l}\text { Embora a maioria das } \\
\text { adolescentes já tenham } \\
\text { ouvido falar dos métodos } \\
\text { contraceptivos, elas não } \\
\text { conheciam lugares nos } \\
\text { quais se sentiriam } \\
\text { confortáveis em acessá- } \\
\text { los. }\end{array}$ \\
\hline $\begin{array}{l}\text { Sserwanj } \\
\text { a, } \\
\text { Musaba } \\
\text { e } \\
\text { Mukuny } \\
\text { a } \\
(2021)\end{array}$ & $\frac{\underline{\text { BMC }}}{\frac{\text { Women's }}{\text { Helath }}}$ & $\begin{array}{l}\text { Estudo } \\
\text { transversal } \\
\text { com } \\
\text { abordagem } \\
\text { quantitativ } \\
\quad \text { a }\end{array}$ & $\begin{array}{l}\text { Participaram do } \\
\text { estudo } 4.264 \\
\text { adolescentes com } \\
\text { idades entre } 15 \text { e } \\
19 \text { anos em } \\
\text { Uganda. }\end{array}$ & $\begin{array}{c}\text { Verificar os } \\
\text { fatores associados } \\
\text { e a prevalência do } \\
\text { uso de } \\
\text { anticoncepcionais } \\
\text { modernos entre } \\
\text { adolescentes entre } \\
15 \text { e } 19 \text { anos do } \\
\text { sexo feminino. }\end{array}$ & $\begin{array}{l}\text { Os resultados mostraram que apenas } 9,4 \% \\
\text { delas utilizavam contraceptivos modernos. } \\
\text { Foi observado que vários fatores } \\
\text { influenciaram a sua utilização. As } \\
\text { adolescentes que eram casadas tinham } \\
60 \% \text { de chances a mais de utilizarem } \\
\text { contracepção em relação as que eram } \\
\text { solteiras, aquelas que haviam engravidado } \\
\text { antes dos } 15 \text { anos eram duas vezes mais } \\
\text { propensas a usarem anticoncepcionais do } \\
\text { que as que engravidaram mais velhas, as } \\
\text { que moravam na região central tinham } \\
95 \% \text { mais chances de usarem contracepção } \\
\text { em relação as que moravam mais a norte, } \\
\text { assim como aquelas que tinham uma } \\
\text { melhor condição socioeconômica eram } \\
91 \% \text { mais propensas a se protegerem. }\end{array}$ & $\begin{array}{l}\text { O estudo mostrou que } \\
\text { fatores como idade da } \\
\text { primeira gestação, apoio } \\
\text { dos parceiros, região } \\
\text { onde habitavam e } \\
\text { condição } \\
\text { socioeconômica } \\
\text { influenciaram } \\
\text { utilização a } \\
\text { contraceptivos entre as } \\
\text { adolescentes em Uganda. }\end{array}$ \\
\hline $\begin{array}{l}\text { Olika, } \\
\text { Kitila, } \\
\text { Terfa e } \\
\text { Olika } \\
(2021)\end{array}$ & $\begin{array}{c}\text { Saúde } \\
\text { Reprodutiva }\end{array}$ & $\begin{array}{l}\text { Pesquisa } \\
\text { demográfic } \\
\text { a e de } \\
\text { saúde com } \\
\text { abordagem } \\
\text { quantitativ } \\
\text { a }\end{array}$ & $\begin{array}{c}\text { Pesquisa realizada } \\
\text { na Etiópica com } \\
\text { quatro rodadas nas } \\
\text { quais } 3710,3266, \\
4009 \text { e } 3381 \\
\text { meninas } \\
\text { participaram } \\
\text { respectivamente }\end{array}$ & $\begin{array}{c}\text { Examinar as } \\
\text { tendências e } \\
\text { correlações do uso } \\
\text { de } \\
\text { anticoncepcionais } \\
\text { entre adolescentes } \\
\text { sexualmente } \\
\text { ativas na Etiópia. }\end{array}$ & $\begin{array}{l}\text { Cerca de } 98 \% \text { das adolescentes tinham } \\
\text { conhecimentos sobre os métodos } \\
\text { contraceptivos. Com isso, foi observado } \\
\text { que o uso de anticoncepcionais aumentou } \\
\text { seis vezes na Etiópia entre } 2005 \text { e } 2016 \text {. O } \\
\text { método mais utilizado foi o injetável, que } \\
\text { aumentou de } 1,6 \% \text { para } 29,1 \% \text { de } 2005 \text { a } \\
\text { 2016. As adolescentes que não tinham } \\
\text { acesso à educação eram de } 90 \% \text { a } 96 \% \\
\text { menos propensas a utilizarem } \\
\text { contracepção. Aquelas com maior poder } \\
\text { aquisitivo tinham três vezes mais chances } \\
\text { de usarem anticoncepcionais, assim como } \\
\text { entre aquelas que tinham acesso as } \\
\text { unidades de saúde, a agentes comunitários } \\
\text { e que se informaram sobre sua saúde } \\
\text { reprodutiva. }\end{array}$ & $\begin{array}{l}\text { Houve um aumento da } \\
\text { utilização de } \\
\text { contraceptivos entre as } \\
\text { adolescentes da Etiópia } \\
\text { nos anos de 2005 a } 2016 . \\
\text { Foi observado uma } \\
\text { mudança no padrão de } \\
\text { anticoncepcionais, } \\
\text { aumentando o uso dos } \\
\text { contraceptivos de longa } \\
\text { duração em relação aos } \\
\text { demais. Fatores } \\
\text { socioeconômicos, } \\
\text { pessoais e de acesso ao } \\
\text { sistema de saúde } \\
\text { influenciaram o uso dos } \\
\text { mesmos. }\end{array}$ \\
\hline
\end{tabular}

Fonte: Autores.

Durante a adolescência, o cérebro tem um expressivo crescimento e desenvolvimento. Apesar de ser um processo rápido, as diferentes partes do encéfalo se modificam em diferentes momentos. Com isso, a parte do sistema nervoso central responsável pela tomada de decisão, pelo planejamento e pensamento abstrato se desenvolvem por último. Dessa forma, há ainda certa imaturidade emocional o que pode ser prejudicial, sobretudo no que tange ao comportamento sexual, que ainda está sendo moldado (Agyemang, et. al., 2019). 
Outro fator a ser observado em adolescentes que fazem uso continuo de anticoncepcional oral, são os efeitos colaterais, que podem ocasionar nas mulheres desde uma dor de cabeça, irritação, vômito, aumento de peso, tonturas, queda de cabeço até uma mudança na libido (Barbosa \& Chaves, 2021).

Tendo isso em vista, o início cada vez mais precoce das atividades sexuais, e a falta de conhecimento acerca dos métodos contraceptivos, são observadas práticas sexuais de forma desprotegida, gerando risco para a transmissão de IST's e a gravidez na adolescência. Dados apontam que apenas 20\% dos homens e 15\% das mulheres utilizaram algum método anticoncepcional na primeira relação sexual, segundos estudos realizados na América Latina (Alves, 2021).

Estudos apontam que a probabilidade de ocorrer o sexo seguro é proporcional com a idade que se possui e está relacionado com fatores socioeconômicos, pois adolescentes mais velhos, na faixa etária dos 18 e 19 anos tendem a utilizar contracepção com mais frequência, assim como aqueles que vivem em áreas urbanas e com acesso à renda familiar, cor/etnia, também são fatores de risco, visto que, jovens autodeclarados pretos/pardos possuem um risco aumentado. Vale ressaltar que o diálogo entre pais/ chefe de família e filhos podem ser condição para o sexo inseguro (Ganle, 2019).

Estudo conduzido entre adolescentes refugiadas de Gana, observou que o planejamento familiar e a escolaridade, tanto das mães, quanto das adolescentes, foram variáveis comuns associadas à prática de sexo transacional, visto que nesse estudo evidenciou-se que $43 \%$ das meninas obtiveram sexo coagido, $36 \%$ sob efeito de substâncias alcoólicas e $71 \%$ tiveram sexo transacional. Entre adolescentes não refugiadas esses números são bem menores. O planejamento familiar e a escolaridade, tanto das mães, quanto das adolescentes, foram variáveis comuns associadas à prática de sexo transacional (Farias, et al., 2016).

Ainda nesse ínterim, a regionalidade consta como outro fator influente. Adolescentes que vivem em áreas urbanizadas e com maior desenvolvimento socioeconômico são mais adeptas ao uso de métodos contraceptivos. A exemplo disso, a região Sul do país é a que possui maior taxa de utilização de métodos contraceptivos, com 42,2\% de adesão, enquanto a região Norte possui uma taxa de apenas 20,4\% de adeptos (Olsen, et al., 2017) Já em Uganda, apenas 9,4\% das jovens habitantes da zona rural faziam uso de algum método contraceptivo moderno. Enquanto isso, mulheres que viviam na parte central, possuíam 93\% de chances a mais de utilização (Bakesiima, et al., 2020).

Em relação ao tipo de método a ser escolhido, o preservativo está em primeiro lugar, dado observado em um estudo realizado na Nigéria, o qual mostrou que das adolescentes solteiras que utilizavam algum método contraceptivo, $75 \%$ delas preferiam o preservativo em relação aos demais, especula-se que isso acontece em razão da facilidade de aquisição e utilização em relação aos outros métodos e a ausência de efeitos colaterais. Logo em seguida, vêm os anticoncepcionais orais. Um dos fatores observados para a baixa aderência aos métodos contraceptivos hormonais foi a forma de utilização. Métodos hormonais de curta duração, como a pílula, demandam regularidade em seu uso, e muitas vezes isso é visto como um obstáculo para sua utilização entre os jovens que informam o esquecimento ou irregularidade na administração do fármaco. Por isso, houve também aumento da procura por métodos de longa duração, com o aumento de 6,4\% do uso de implantes (Rosaneli, et al., 2020).

Em uma pesquisa realizada em Gana, $90 \%$ das adolescentes conheciam e eram capazes de citar pelo menos um método contraceptivo, sendo o principal o preservativo. Apesar disso, $82 \%$ relataram não fazer uso de nenhum método. A principal causa apontada foi a possibilidade de ocorrência de efeitos colaterais, e uma pequena parcela de $3 \%$ afirmou ser a causa a inconveniência atribuída ao uso (Paiva, et. al., 2008).

Sobre a anticoncepção de emergência - AE, preocupa o fato do conhecimento das adolescentes por vezes se restringirem à existência do fármaco, mas não sobre o uso correto e efeitos colaterais. O estudo mostrou que o uso da $\mathrm{AE}$ de forma regular, fato extremamente preocupante, em razão não só dos efeitos adversos, mas também da falha na prevenção de 
IST's. Vale mencionar que a distribuição desse método é amplamente irregular no sistema de saúde, variando a depender da localidade (Sousa, et. al., 2006).

Acerca dos efeitos colaterais citados, em um estudo alemão foi constatado que o uso de anticoncepcionais orais não está relacionado como causa de alteração da saúde mental, no entanto, está ligado a maior probabilidade de uso de psicotrópicos e antidepressivos, além de elevação da pressão arterial e dos níveis séricos de vitamina D, o que pode assustar os adolescentes. Ademais, o aumento do peso, acne, risco de trombose venosa profunda e outros, são fatores que também pesam na escolha do método contraceptivo (Lewandowski, et. al., 2020).

Um dos fatores que influenciou a utilização ou não de métodos contraceptivos foi a escolaridade. As adolescentes sexualmente ativas sem acesso à educação formal e ensino fundamental são cerca de $96 \%$ menos propensas a usarem algum anticoncepcional. Assim como aquelas que visitaram a unidade básica de saúde tinham três vezes mais chances de adotarem algum desses métodos em comparação aquelas que não haviam sido instruídas sobre o período fértil (Olika, et. al., 2021).

Assim, fica evidente que esse ato educativo apresenta falhas, inclusive na esfera familiar. Alguns pais podem ter uma percepção e atitude negativa para conversar sobre sexo. Isso está relacionado com os valores inerentes a cada geração. Muitas vezes ao apresentar o tema aos filhos, eles podem sentir que estão corrompendo a inocência do adolescente ou incentivando a promiscuidade, entendem como um incentivo ao início da prática sexual. Frequentemente nem mesmo os pais receberam a educação adequada sobre isso (Crawford, et. al., 2021).

Culturalmente, ainda existe o "tabu social" que a busca por contraceptivos apresenta. Em muitos locais, mulheres que fazem sexo sem estar em um relacionamento são consideradas promíscuas. Em outros, o uso é inadequado pois tem como consequência a redução da população. Religiosamente, a prática sexual antes do casamento é considerada um pecado, contribuindo grandemente com a baixa adesão ao uso de anticoncepcionais em regiões/famílias muito religiosas (Medeiros, et. al., 2016; Sousa, et. al., 2006).

A desigualdade de gênero que ainda permeia a sociedade acaba sendo um entrave para a anticoncepção. Em um primeiro cenário, homens podem ser contra ao uso de métodos contraceptivos por acreditarem que estes abririam um precedente para a promiscuidade ou infidelidade. Em outro há o fato que grande parte das ações educativas são voltadas apenas para mulheres, o que torna importante a inclusão desse público em ações coletivas de saúde.

Além disso, frequentemente a criação e formação da identidade sexual feminina pode ocorrer de forma rígida e repressiva, associada ao estereótipo de gênero de promiscuidade quando mais de um parceiro sexual. A falta de liberdade na escolha do método contraceptivo pode culminar na medicalização do corpo feminino, como o uso de um método a que elas não se sintam confortáveis ou mesmo a realização de procedimentos invasivos, como a laqueadura (Olsen, et. al., 2017).

$\mathrm{Na}$ Nigéria, as fontes de informação mais importantes dos entrevistados sobre contracepção incluíram televisão (41,7\%), rádio (27,6\%) e em último lugar, profissionais de saúde (11,5\%). Apesar de 38,5\% das entrevistadas que tinham ouvido falar sobre anticoncepcionais não conheciam nenhum local específico onde pudessem obter anticoncepcionais (Leite, et al., 2020).

O despreparo de alguns profissionais de saúde, por vezes, sobrepõe crenças pessoais e agem de modo incisivo, afastando o adolescente. Ademais, a falta de um espaço físico seguro para acolher os adolescentes, que garanta o sigilo e conte com ajuda profissional qualificada. E por fim, o baixo investimento em políticas públicas para a educação e conscientização dos adolescentes (Portela \& Araújo, 2013).

Um estudo realizado na Etiópia mostra significativo aumento do uso de métodos contraceptivos. $\mathrm{O}$ uso de contraceptivos por adolescentes sexualmente ativas cresceu 32,7\% entre os anos de 2000 a 2016. Além disso, houve aumento também no uso de anticoncepcionais modernos, notadamente o implante intradérmico e injetáveis. Isso pode ser atribuído para 
um conjunto de intervenções que foram implantadas pelos serviços de saúde, a nível governamental e privado (Cardoso, et, al., 2019).

Com isso, mesmo que tenha ocorrido um aumento na taxa de utilização dos métodos contraceptivos entre os adolescentes, muitos ainda não são adeptos aos anticoncepcionais e muitos são os fatores que influenciam essa prática, como a falta de escolaridade, pressão do parceiro ou o acesso ao sistema de saúde (Araújo, 2015).

\section{Conclusão}

Com isso, conclui-se que apesar do aumento na taxa de adesão aos métodos contraceptivos entre os adolescentes, muitos desses ainda não os utilizam, aumentando o risco de infecções sexualmente transmissíveis e gravidez na adolescência.

São diversos os fatores que influenciam tal prática, sendo os mais prevalentes a dificuldade de acesso a educação e ao sistema de saúde, a falta de diálogo com os pais ou familiares, a vulnerabilidade social, a faixa etária e a desigualdade de gênero.

Verificou-se a importância da educação em saúde voltada para a saúde reprodutiva desses jovens, visto que aqueles com maior escolaridade e acesso aos meios de comunicação tinham mais chances de adotarem uma contracepção.

Além disso, mesmo o preservativo sendo ainda o método contraceptivo mais conhecido, foi observado uma mudança no padrão de escolha dos métodos. Os contraceptivos escolhidos tendiam a ser os de curta duração, como os anticoncepcionais orais, porém atualmente vem crescendo a adesão aos métodos de longa duração como os implantes e o DIU.

Dessa forma, fica evidente a necessidade de melhoria e ampliação das medidas públicas de saúde voltadas para a sexualidade do adolescente, visando aumentar a taxa de adesão aos métodos contraceptivos e a melhoria da qualidade de vida dos mesmos, assim como pesquisas que fundamente a temática.

\section{Referências}

Agyemang, J., Newton, S., Nkrumah, I., Tsoka-Gwegweni, J. M. \& Cumber, S. N. (2019) Contraceptive use and associated factors among sexually active female adolescents in Atwima Kwanwoma District, Ashanti region-Ghana. Pan African Medical Journal. DOI: https://doi.org/10.11604/pamj.2019.32.182.15344.

Alves, A. S. \& Lopes, M. H. B. M. (2008). Uso de métodos anticoncepcionais entre adolescentes universitários. Rev Bras de Enferm, 61(2). https://doi.org/10.1590/S0034-71672008000200005.

Alves, J. D. S. A., Gama, S. G. N. D., Viana, M. C. M., Martinelli, K. G. \& Neto, E. T. D. S. (2021) Características socioeconômicas influenciam as atitudes face à sexualidade em adolescentes. J Hum Growth Dev. https://doi.org/10.36311/jhgd.v31.11084.

Araújo, A. V. S. D. (2015). O papel dos pais na educação sexual de adolescentes: uma revisão integrativa. Revista eletrônica da Universidade Vale do Rio Verde. https://doi.org/10.5892/ruvrd.v13i1. 2176.

Bakesiima, R., Cleeve, A., Larsson, E., Tumwine, J. K., Ndeezi, G., Danielsson, K. G., Nabirye, R. C. \& Kashesya, J. B. (2020). Modern contraceptive use among female refugee adolescents in northern Uganda: prevalence and associate factors. Reproductive Health. https://doi.org/10.1186/s12978-020-00921-y.

Barbosa, A. S., \& Chaves, C. T. de O. P. (2021). Consequências do uso contínuo de anticoncepcional: um alerta as mulheres. Research, Society and Development, 10(15), e349101522949. https://doi.org/10.33448/rsd-v10i15.22949.

Brasil. (2019). Lei 8.069, de 13 de julho de 1990. Estatuto da Criança e do Adolescente. https://www.planalto.gov.br/ccivil_03/leis/18069.htm.

Campos Júnior, D \& Burns, D. A. R. (2014). Tratado de pediatria. Manole.

Cardoso, L. C. D. S., Bendi, A. L., Santos, L. T. V. D. S., Lima, B. L. D. O. \& Einioft, M. Souza, A. (2019). A utilização de contraceptivos hormonais por adolescentes e potenciais riscos para a saúde. Clin Biomed Res. https://doi.org/10.4322/2357-9730.85153.

Costa, I. G. D. D. \& Carvalho, A. A. (2014). Uso de contracepção por mulheres de diferentes grupos religiosos: diferenças ou semelhanças? Dossiê: Religião e Demografia. https://doi.org/10.5752/P.2175-5841.2014v12n36p1114.

Crawford, E. E., Atchison, C. J., Ajayi, Y. P. \& Doyle, A. M. (2021). Modern contraceptive use among unmarried girls aged 15-19 years in South Western Nigeria: results from a cross-sectional baseline survey for the Adolescent 360 (A360) impact evaluation. Reprod Health. https://doi.org/10.1186/s12978-02001056-w.

Dadoorian, D. (2003). Gravidez na adolescência: um novo olhar. Psicol cienc. https://doi.org/10.1590/S1414-98932003000100012. 
Ezenwak, U., Mbachu C., Ezumah, N.,, Eze, I., Agu, C., Agu, I. \& Onwujekwe, O. (2020). Exploring factors constraining utilization of contraceptive services among adolescents in Southeast Nigeria: an application of the socio-ecological model. BMC Public Health. https://doi.org/10.1186/s12889-020-09276-2.

Farias, M. R., Leitel, S. N., Tavaresll, U. L., Oliveira, M. A., Arrais, P. S. D., Bertoldi, A. D., Pizzol, T. S. D., Luiza, V. L., Ramos, L. R. \& Mengue, S. S. (2016). Utilização e acesso a contraceptivos orais e injetáveis no Brasil. Rev Saúde Pública. https://doi.org/10.1590/S1518-8787.2016050006176.

Ganle, J. K., Amoako, D., Baatiema, L. \& Ibrahim, M. (2019) Risky sexual behaviour and contraceptive use in contexts of displacement: insights from a crosssectional survey of female adolescent refugees in Ghana. Int J Equity Health. https://doi.org/10.1186/s12939-019-1031-1.

Leite, A. A. G. R., Passos, A. L. V., Araújo, L. F. D., Negreiros, F \& Freire, S. E. D. A. (2020). Conhecimentos, práticas e atitudes frente à anticoncepção de emergência: revisão sistemática. Psic. Rev. São Paulo. https://doi.org/10.23925/2594-3871.2020v29i1p201-222.

Lewandowski, S. K., Duttge, G. \& Meyer, T. (2020) Quality of life and mental health in adolescent users of oral contraceptives. Results from the nationwide, representative German Health Interview and Examination Survey for Children and Adolescents. Quality of Life Research. https://doi.org/10.1007/s11136-020$02456-y$.

Medeiros, T. F. R. D., Santos, S. M. P. D., Xavier, A. G., Gonçalves, R. L., Mariz, S. R. \& Souza, F. L. P. D. (2016). Vivência de mulheres sobre contracepção na perspectiva de gênero. Rev Gaúcha Enferm. https://doi.org/10.1590/1983-1447.2016.02.57350.

Olika, A. K., Kitila, S. B., Terfa, Y. B. \& Olika, A. K. (2021). Contraceptive use among sexually active female adolescents in Ethiopia: trends and determinants from national demographic and health surveys. Reprod Health. https://doi.org/10.1186/s12978-021-01161-4.

Olsen, J. M., Lago, T. D. G., Kalckmann, S., Alves, M. C. G. P. \& Escuder, M. M. L. (2017) Práticas contraceptivas de mulheres jovens: inquérito domiciliar no Município de São Paulo, Brasil. Cad. Saúde Pública. https://doi.org/10.1590/0102-311X00019617.

Olsen, J. M., Lago, T. D. G., Kalckmann, S., Alves, M. C. G. P. \& Escuder, M. M. L. (2017). Práticas contraceptivas de mulheres jovens: inquérito domiciliar no Município de São Paulo, Brasil. Cad. Saúde Pública. https://doi.org/10.1590/0102-311X00019617.

Paiva, V., Calazans, G., Venturi, G. \& Dias, R. (2008). Idade e uso de preservativo na iniciação sexual de adolescentes brasileiros. Ver Saúde Pública. https://doi.org/10.1590/S0034-89102008000800007.

Portela, N. L. C. \& Araújo, L. P. D. (2013). Conhecimento e prática dos métodos contraceptivos por estudantes adolescentes: um estudo comparativo. Revista Univap. https://doi.org/10.18066/revunivap.v19i33.130.

Rosaneli, C. F., Costa, B. C. \& Sutille, V. M. (2020). Proteção à vida e à saúde na gravidez na adolescência sob o olhar da bioética. Revista de Saúde Coletiva. https://doi.org/10.1590/S0103-73312020300114.

Sousa, L. B. D., Fernandes, J. F. P. \& Barroso, M. G. T. (2006). Sexualidade na adolescência: análise da influência de fatores culturais presentes no contexto familiar. Acta Paulista de Enfermagem. https://doi.org/10.1590/S0103-21002006000400007.

Souza, M. T., Silva, M. D., \& Carvalho, R. (2010). Revisão integrativa: o que é e como fazer. einstein (São Paulo), 8(1), 102-106. https://doi.org/10.1590/s1679-45082010rw1134.

Sserwanja, Q., Musaba, M. W. \& Mukunya, D. (2021). Prevalence and factors associated with modern contraceptives utilization among female adolescents in Uganda. BMC Women's Health. https://doi.org/10.1186/s12905-021-01206-7.

U.S. Department of Health and Human Services, Office of Adolescent Health, Adolescent Development Explained, Washington, D.C: U,S. Government Printing Office (2018). https://www.planalto.gov.br/ccivil_03/leis/18069.htm.

Vieira, L. M., Saes, S. O., Dória, A. A. B. \& Goldberg, T. B. L. (2006) Reflexões sobre a anticoncepção na adolescência no Brasil. Ver Bras Saude Mater Infant. https://doi.org/10.1590/S1519-38292006000100016.b. 